\title{
Overview of the New European Federation of Neurological Societies' Guidelines on Molecular Diagnosis of Neurological Disorders
}

\author{
Jean-Marc Burgunder, ${ }^{1}$ Thomas Gasser, ${ }^{2}$ Hanne $\mathrm{F} \mathrm{Harbo}{ }^{3}$ and Josef Finsterer ${ }^{4}$
}

\begin{abstract}
1. Professor of Experimental Neurology, Department of Neurology, University of Bern; 2. Professor of Neurology, Department of Neurodegenerative Diseases, Hertie-Institute for Clinical Brain Research, University of Tübingen; 3. Professor of Neurology, Department of Neurology, Oslo University Hospital, Ullevål and University of Oslo; 4. Professor of Neurology, Department of Neurology, KA Rudolfstiftung, Vienna and Danube University Krems
\end{abstract}

\section{Abstract}

There has been an explosion of knowledge concerning genetic causation of a number of neurological disorders in the last couple of years since the discovery of the gene mutated in Huntington's disease almost 20 years ago. This has led to the difficulty of making appropriate choice in the use of genetic testing during the diagnostic procedures in such cases. Therefore the European Federation of Neurological Societies (EFNS) has set up a task force to develop guidelines for the general neurologist, and a new series of largely expanded papers has now been published covering the major areas of the field. In this article, the major suggestions established in these guidelines after systematic review of the evidence at hand and experts reaching a consensus are summarised. Currently, there are a number of instances in which molecular testing is of great practical help; however, some instances remain where the place of such a diagnostic tool is not established.
\end{abstract}

\section{Keywords}

Genetic testing, guideline, hereditary disorder

Disclosure: The authors have no conflicts of interest to declare.

Received: 4 October 2010 Accepted: 19 November 2010 Citation: European Neurological Review, 2010;5(2):12-7 DOI:10.17925/ENR.2010.05.02.12

Correspondence: Jean-Marc Burgunder, Department of Neurology, University of Bern, Inselspital, CH-3010 Bern, Switzerland. E: Jean-marc.burgunder@dkf.unibe.ch

Knowledge on the genetic background of a number of neurological disorders has tremendously increased in the last years. The European Federation of Neurological Societies (EFNS) published guidelines for genetic testing in clinical practice in 2001 in two successive papers. ${ }^{1,2}$ A new EFNS task force has now revised the guidelines about genetic testing in the major neurogenetic disorders in five paper ${ }^{3-7}$ and a summary of the findings is given here. The task force had taken a consensus search strategy based on a thorough analysis of published data and circulation of the proposals until general agreement amongst the members was achieved. EFNS has set general criteria to assess evidence for management in neurological disorders ${ }^{8}$ and the task force has used the levels of evidence gradation presented as a frame for future EFNS guidelines and adapted them to genetic testing. The gold standard for genetic diagnostics is the confirmation of a particular mutation established to be causative for the specific disorder under consideration. In this perspective such a test cannot be compared with another diagnostic test to assess its accuracy. Quality of the studies used for guideline development has been taken into consideration in order to rate the level of recommendation. In the absence of prospective studies in the strict sense, they have included retrospective studies (class II and III studies according to the guidelines for preparation of neurological guidelines ${ }^{8}$ ) and not blinded studies and expert opinions (class IV). In a number of instances, level B (convincing class II or overwhelming class III evidence ${ }^{8}$ ) and level $\mathrm{C}$ ratings (at least two convincing class III studies) could be established, in other instances only expert opinions from the panel members could be offered, while in rare disorders the evidence data at hand did not allow any generalised recommendation. Disorders, for which the available data allow a rating at all, have been summarised in Table 1.

\section{Huntington's Disease}

Huntington's disease $(\mathrm{HD})^{9}$ was the first major neurogenetic autosomal dominant disorder for which the gene mutation causing the disease was identified. ${ }^{10}$ Expansion of a cytosine-adenine-guanine (CAG)-triplet in exon 1 of the HTT gene (formerly called HD or IT15) causes the clinical syndrome characterised by choreatic movements and cognitive and neuropsychiatric disturbances. Normally, CAG repeat numbers range from 10 to 26 , but in $\mathrm{HD}$ the repeat number is from 36 to 121. The CAG expansion leads to the formation of an elongated polyglutamine (polyQ) sequence within the encoded protein, huntingtin. ${ }^{10}$ The lowest repeat numbers are seen in adult onset patients. CAG repeats above 40 are usually penetrant, and young-onset patients often have more than 60 CAG repeats.

Diagnostic testing for HD is recommended when patients present with neurological signs and symptoms of the disease (see Table 1) whether or not they have a family history. Informed consent and adequate genetic counselling is particularly important in these situations. The diagnosis may have implications for family members, and previously established guidelines for pre-symptomatic molecular diagnosis should be followed. ${ }^{11}$ A clinical picture with similarity to HD may be caused by mutations in other genes; however, no general recommendations can be given for testing of less-frequent mutations causing HD. ${ }^{12}$

\section{Parkinson's Disease}

Point mutations, ${ }^{13}$ but also duplications and triplications ${ }^{14,15}$ of the gene for alpha-synuclein (SNCA), can cause an autosomal-dominant form of Parkinson's disease (PD). ${ }^{16}$ Point mutations and triplications 
are rare ${ }^{17,18}$ and have mostly been found in cases with a strong dominant family history and a high prevalence of dementia, but SNCA-duplications can cause late-onset typical PD. In Europeans, mutations in the LRRK2 gene are a much more common cause of dominant PD, and thus molecular testing for LRRK2 is recommended in familial cases with dominant inheritance. ${ }^{19,20}$ Testing for the LRRK2 G2019S mutation is recommended in familial and also sporadic patients in specific populations, e.g. in the Ashkenazim or North African Arabs. ${ }^{21}$ Testing for mutations in recessive PD-genes (parkin, PINK1, DJ-1) is recommended in families suggestive of recessive inheritance (affected sibling pairs) or sporadic patients with very early onset $<35$ years $^{22}$ (see Table 1).

\section{Dystonia and Other Movement Disorders}

No genetic tests can be recommended in the more common focal dystonias, and molecular diagnosis of this phenotype is still restricted to a relatively small proportion with a clearly defined familial disease..$^{23}$ The major cause of early-onset generalised dystonia is a deletion of the trinucleotide GAG (encoding glutamic acid) in the gene for torsinA on chromosome 9 q34 (DYT1). ${ }^{24}$ More than $50 \%$ of patients with this phenotype have this mutation. Due to the reduced penetrance of the GAG-deletion, DYT1-mutation carriers often have a negative family history. Molecular testing for this GAG-deletion is recommended (see Table 1) in patients with early- (<26 years) and limb-onset generalised dystonia, regardless of their family history. ${ }^{25}$

Early-onset generalised dystonia with a clear response to levodopa (levodopa-responsive dystonia) is most commonly found to be caused by point mutations or exon deletions in the gene encoding GTP-cyclohydrolase-1 (GCH1). ${ }^{26}$ Testing for GCH1-mutations including gene dosage studies is recommended in these patients. In patients with a typical clinical picture and a family history suggestive of myoclonus-dystonia syndrome, sequencing and gene dosage studies of the SGCE-gene ${ }^{27}$ are recommended. Sequencing of the ATP7B gene can confirm a diagnosis of Wilson disease for family counselling purposes. ${ }^{28}$ However, blood and urine chemistry, particularly copper excretion in urine and serum coeruloplasmin levels, is still the diagnostic method of choice in the majority of Wilson cases.

\section{Ataxias}

In patients with an adult onset of slowly progressive ataxia and a family history compatible with autosomal dominant inheritance, genetic testing should include SCA1, 2, 3, 6, 7 and 17, which represent the majority of the presently identified genotypes for one of the spinocerebellar ataxias in Europe. ${ }^{29-32}$ Among those, priorities should be chosen according to associated clinical features: a pure cerebellar syndrome occurs in SCA6, while SCA1, 2 and 3 patients show variable involvement of the extrapyramidal, pyramidal and peripheral nervous system (see Table 1). Cases with SCA7 are typically distinguished by retinal degeneration. In male patients with late-onset ataxia, particularly if combined with a mixed tremor, extrapyramidal symptoms and mild cognitive deterioration, testing for the fragile X-tremor-ataxia syndrome (FXTAS), which is caused by a pre-mutation in the FMR1 gene (a CGG-trinucleotide-repeat, expanded to a size of 55 to 200 repeats), is recommended. A multitude of different recessive syndromes with progressive ataxia as the clinical core symptom have been recognised. ${ }^{33}$ Most cases have onset in childhood or early adulthood, but late-onset cases are increasingly recognised and should be considered in the differential diagnosis of any ataxic patient. In cases presenting with a combination of early-onset ataxia, peripheral sensory neuropathy and absence of marked cerebellar atrophy on magnetic resonance imaging (MRI), genetic tests for the most common form of recessive ataxia, Friedreich's ataxia (FRDA) is recommended. ${ }^{34}$ The disease is caused by an easily detectable trinucleotide repeat (GAA) in intron 1 of the FRDA gene. ${ }^{33}$ Genetic testing can be helpful in several other rare recessive forms of ataxia. Molecular testing for ataxia telangiectasia (ATM), ${ }^{35}$ ataxia with oculomotor apraxia (AOA1 and $A O A 2)^{36}$ is recommended when guided by reduced levels of albumin or increased levels of cholesterol (in AOA) or elevated alpha-fetoprotein (in ATM), even in the absence of conspicuous clinical features. Ataxia with vitamin E deficiency (AVED) can usually be diagnosed reliably by measuring vitamin $E$ levels in serum. However, molecular diagnosis can be helpful for early detection in siblings of patients with an established diagnosis, and thus may help to institute early nutritional therapy. In the rare patient with clearly distinct attacks of ataxia and vertigo, a form of the episodic ataxias can be suspected. Genetic testing for EA1 and EA2 is available in some specialised laboratories.

\section{Spastic Paraplegia}

The hereditary spastic paraplegias (HSPS) are characterised by slowly progressive pyramidal tract dysfunction occurring in either 'pure' or 'complicated' forms, the latter being associated with various other neurologic signs and symptoms. ${ }^{37}$ To date, about 40 genetically distinct forms are recognised, and in almost half of them the genes have been identified. There are about as many autosomal dominant (AD) as recessive (AR) forms, in addition to at least 3 X-linked HSPS. Most of the AD-HSPs present clinically as pure HSP, while most of the AR-HSPS are complicated forms.

Patients with pure HSP and a family history of spastic paraparesis should be tested for point mutations in the SPAST gene (SPG4), which account for almost $50 \%$ of these cases (see Table 1). ${ }^{38}$ If direct sequencing of the gene is negative, a multiplex ligation-dependent probe amplification assay (MLPA) should be applied to identify genomic rearrangements. As a third step, sequencing of atlastin $(\mathrm{SPG})^{39}$ in subjects with a pure form and onset under 20 years is recommended. All other forms are very rare. Sequencing of REEP1 and KIF5A can be considered in remaining mutation-negative dominant families, the latter particularly when a neuropathy is present. If the pedigree analysis suggests an X-linked form, testing for mutations in L1-CAM and PLP (SPG 1 and SPG2) should be proposed in early onset complex forms of HSP with white-matter changes on MRI. ${ }^{40}$ In putatively recessive cases, molecular testing first for SPG11 and second for SPG15 is recommended in patients with a thin corpus callosum. SPG7 should be tested, especially when cerebellar features are present. For other recessive and X-linked forms of HSP no general recommendation can be given. Subjects with sporadic progressive spastic paraparesis should be tested for SPG4 mutations by MLPA after careful exclusion of other causes of spasticity. In negative cases, sequencing of SPG7 can be proposed.

\section{Dementia}

Inherited degenerative dementias occur in variable proportion of the cohorts, most often as autosomal-dominant disorders and have similar phenotypes to the sporadic disease. Particular attention needs to be given to adequate genetic counselling and consenting from the patient and/or family caregiver prior to any attempt of molecular genetic diagnosis in dementing disorders. ${ }^{41}$ In most instances, 
Table 1: Genetic Tests Suggested to be Used in Clinical Neurology Practice

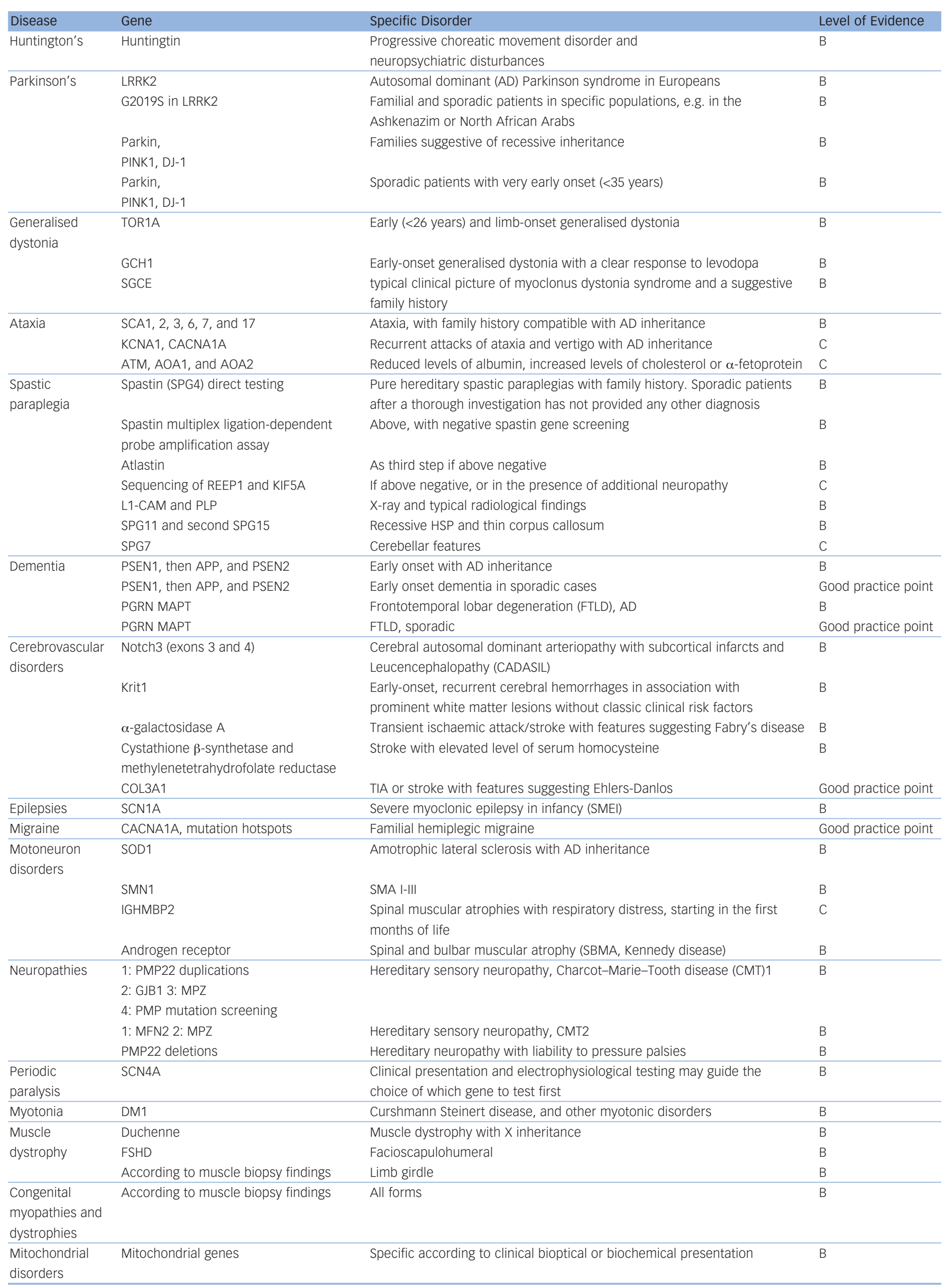


molecular genetic diagnosis will only be feasible in cases with a clear positive family history that is indicative of a monogenic form of the disease or in sporadic cases with an unusually early age of onset. ${ }^{42}$ In families with an autosomal dominant occurrence of Alzheimer's disease, mutational screening can be performed first in presenilin 1 (PSEN1), then in the amyloid precursor protein gene (APP) and finally (if negative) in presenilin 2 (PSEN2), which can be useful for genetic counselling in cases of clearly dominantly inherited $A D^{43}$ (see Table 1). In younger patients with sporadic disease, genetic screening can be also considered. In cases with frontotemporal dementia with autosomal dominant inheritance, genetic testing for mutations in the progranulin (PRGN) ${ }^{44}$ and MAPTau (MAPT) $)^{45}$ genes is recommended Testing can also be considered in sporadic cases, although mutations are found only in less than $5 \%$. Numerous mutations in the prion protein gene have been found in familial cases of prion disorders, occurring in about $10-20 \%$ of cases. Special laboratories offer full sequencing of the gene in search for mutations.

\section{Cerebrovascular Disorders}

While the genetic background of cerebrovascular disease in general is well recognised and a number of susceptibility genes have been proposed, most cases of stroke do not have a single genetic cause..$^{46,47}$ Early-onset, familial occurrence and specific features may lead to the suspicion of a Mendelian inherited stroke, which can be confirmed by the finding of a mutation (see Table 1). For example, migraine with aura in the third decade of life followed by recurrent ischaemic stroke and dementia with a typical MRI picture is found in cerebral autosomal dominant arteriopathy with sub-cortical infarcts and leukencephalopathy (CADASIL) resulting from mutations in the gene for Notch3 on chromosome 19q12. ${ }^{48}$ Diagnosis is supported by typical osmiophilic granula found in a skin biopsy. Mitochondrial disorders are discussed below. Cerebral amyloid angiopathy exists in different forms and is due to mutations in the amyloid precursor gene or the cystatin gene. ${ }^{49}$

In half of the cases of cerebral cavernous malformations, which may lead to intracerebral or subarachnoid bleeding and autosomal dominant inheritance with incomplete penetrance is found. The most frequent mutations are found in the KRIT1 gene. ${ }^{50}$ Genetic testing may be important to assess risk in family members; however, this can also be performed by MRI.

\section{Episodic Disorders}

A number of neurological episodic disorders has now been characterised at the molecular level, many related to mutations in genes encoding ion channels (i.e. chanellopathies). Usually the diagnosis can be made on clinical and electrophysiological grounds, and the usefulness of molecular diagnosis outside of research protocols is debated, especially since in a number of them the genes involved are quite large and mutations found not only on hot spots. Muscle channelopathies include myotonia, paramyotonia and periodic paralysis due to mutations in the muscle chloride, sodium, potassium or calcium channel. ${ }^{51}$

In rare cases of epilepsy, an autosomal dominant or recessive inheritance pattern can be recognised and many of the genes involved in such cases also encode ion channels. ${ }^{52} \mathrm{~A}$ high level of suspicion is needed to suspect severe myoclonic epilepsy of infancy (SMEI), and perform a search for mutations in the sodium channel gene SCN1A in order to adequately manage these patients. ${ }^{53}$
Familial hemiplegic migraine ${ }^{54}$ is a rare inherited form of the disorder and may be due to mutations in the alpha1 subunit of the calcium channel gene (CACNA1A), less frequently in the ATP1A2 or in the neuronal voltage-gated sodium channel gene (SCN1A).

\section{Neuropathies and Motor Neuron Disorders}

The first step in finding the aetiology of a hereditary neuropathy, which together represent the most common inherited neuromuscular disorder ${ }^{55}$ is a precise clinical and physiological work up. ${ }^{6}$ According to these findings hereditary neuropathies are classified into hereditary sensory-motor neuropathies (HSMN, Charcot-Marie-Tooth disease $[\mathrm{CMT}]$ ), hereditary sensory and autonomous neuropathies, hereditary motor neuropathies and the familial brachial plexus neuropathy. According to nerve conduction studies and the mode of inheritance HSMN are divided into the autosomal dominant CMT1 (nerve conduction velocity $<38 \mathrm{~m} /$ second) and CMT2 (reduced compound muscle action potential amplitude but normal or only slightly decreased conduction velocity) and the autosomal recessive CMT4. Further classification can be performed according to the inheritance pattern. In autosomal dominant cases, ${ }^{56}$ the search for the causative mutation is best performed by selecting the gene according to the frequency of occurrence in a particular type of hereditary neuropathy (see Table 1). In CMT1 a PMP22 duplication ${ }^{57}$ should be excluded first. If it cannot be found, GJB1 and then MPZ should be tested sequentially. In CMT2, MFN2 is the first gene to be screened, followed by MPZ and GJB1. Hereditary neuropathy with liability to pressure is usually due to a PMP22 deletion, which can be readily assessed. Other forms of hereditary neuropathies that exist, including motor neuropathy and sensory-autonomous neuropathy are rarer and diagnostic test is often not yet readily available.

Amyotrophic lateral sclerosis is inherited in $5-10 \%$ of cases. Most frequently, mutations in the SOD1 gene can be found..$^{58}$ In spinal muscular atrophy a homozygous deletion of exon 7 of the telomeric copy of the survival motor neuron gene (SMN1) is most frequently found and searching for this mutation is recommended for diagnosis and counselling (see Table 1). If bulbospinal muscular atrophy is suspected it should be tested for a CAG-repeat expansion $>40$ in the adroegen receptor gene.

\section{Myopathies}

In recent years a tremendous increase in our knowledge on hereditary myopathies has been achieved making the diagnostic process much more complex, but also allowing a more precise classification regarding diagnosis and prognosis. Some myopathies can already be readily classified according to the phenotype. If the phenotype suggests a muscular dystrophy with $\mathrm{x}$ chromosomal inheritance, the most probable diagnosis is Duchenne muscular dystrophy ${ }^{59}$ or another form of dystrophinopathy and a molecular diagnostic test can be performed (see Table 1). In case of an autosomal dominant muscular dystrophy with involvement of the face and asymmetric atrophy of the shoulder and proximal arm muscles, a genetic test for facio-scapulo-humeral dystrophy ${ }^{60}$ can be performed. If the phenotype suggests myotonic dystrophy $1^{61,62}$ search for a CTG-triplet repeat expansion in the DMPK gene can be performed without the need for any additional diagnostic test. In most other cases, muscle biopsy retains an important role for the complementary phenotypical classification. Histological examination allows a morphological diagnosis, including a number of sporadic or metabolic myopathies, as well as a classification as a dystrophy or congenital myopathy, for 
example. Further studies of protein expression using the same material examined by immunohistochemistry or immunoblotting may allow a further delineation of the protein most probably involved in the process, for example in differentiating between the numerous forms of limb girdle muscle dystrophies. ${ }^{62}$ This allows the proper choice of the molecular test. Many more rare myopathies can be studied in a research setting for which no routine molecular testing is yet available. However, in many of these cases a morphological diagnosis might be sufficient.

\section{Mitochondrial Disorders}

Mitochondrial disorders (MIDS) are increasingly recognised and since the diagnostic facilities become more and more sophisticated and more widely available, their prevalence is also mounting. ${ }^{6}$ Confirmation of the genetic defect is the golden standard of diagnosing these conditions but compounded by the possibility that either the mitochondrial (mtDNA) or nuclear genome (nDNA) or both genomes simultaneously carry a mutation, that syndromic and non-syndromic phenotypes exist, and that not only defects of the respiratory chain (RC) or oxidative phosphorylation (OXPHOS) cause a MID but also defects in the $\beta$-oxidation, hem-metabolism or transport machinery ${ }^{64}$ To limit the expanding field to the most relevant and frequent disorders the EFNS-guidelines ${ }^{5}$ were restricted to MIDs due to RC/OXPHOS defects.

RC/OXPHOS-MIDS may be classified into those resulting from an mtDNA mutation or those resulting from an nDNA mutation. Due to the peculiarities of the mtDNA a mutation may affect all copies of a cell or tissue (homoplasmy) or only a portion of them (heteroplasmy). ${ }^{65}$ Homoplasmic mutations are regarded as tissue-specific, whereas a heteroplasmic mutation occurs in all tissues, but this view has been recently challenged. mtDNA mutations may be due to deletions (rearrangements), such as in Kearns Sayre syndrome (KSS), ${ }^{66,67}$ progressive external ophthalmoplegia (PEO), ${ }^{67}$ Pearson syndrome ${ }^{68}$ or non-syndromic MIDs. They may be also caused by point mutations in genes encoding either proteins, as in Leber hereditary optic neuropathy (LHON), ${ }^{69}$ neuropathy, ataxia and retinitis pigmentosa (NARP). ${ }^{70}$ maternally inherited Leigh syndrome (MILS) ${ }_{i}^{\cdot 71}$ tRNAS as in mitochondrial encephalopathy with lactate acidosis (MELAS), ${ }^{72}$ myoclonus epilepsy with ragged red fibres (MERRF) ${ }^{73}$ and non-syndromic MIDs or rRNAs as in non-syndromic deafness.

Syndromic MIDs due to nDNA mutations include Leigh syndrome, Leigh-like syndrome, Barth syndrome, autosomal dominant or recessive $\mathrm{PEO}$, myo-neuro-gastro-intestinal encephalomyopathy $(\mathrm{MNGIE}){ }^{74}$ dominant optic atrophy with deafness (ADOAD), ${ }^{75}$ ataxia-neuropathy spectrum (ANS), ${ }^{76}$ optic atrophy, myoclonic epilepsy, myopathy, sensory ataxia (MEMSA), growth retardation, aminoaciduria, cholestasis, iron overload, lactacidosis and early death (GRACILE), Alpers-Huttenlocher syndrome (AHS) mtDNA breakage syndromes and depletion syndromes. However, the majority of the nDNA-encoded MIDs present as non-syndromic encephalopathies, encephalomyopathies or encephalocardiomyopathies. MIDs due to nDNA gene mutations may be classified into those, which result from mutated RC-subunits, ancillary proteins involved in the formation, turnover or function of RC-complexes (RCCS), proteins involved in the maintenance of the mtDNA, resulting in breakage syndromes (multiple deletions) or depletion syndromes, proteins involved in the mitochondrial protein synthesis machinery (translation disorders), proteins involved in the maintenance of the lipid-milieu, proteins involved in the CoQ production, proteins involved in the mitochondrial transport machinery or mitochondrial biogenesis, or proteins involved in apoptosis.

The genetic diagnosis of a MID is based on a comprehensive individual and family history and clinical and instrumental investigations by neurologists, ophthalmologists, otologists, endocrinologists, cardiologists, gastroenterologists, nephrologists, hematologists, radiologists or dermatologists. If biochemical investigations of biopsies from the muscle or other affected tissues support the clinical suspicion of a sporadic or inherited syndromic or non-syndromic MID, genetic testing is indicated. If the phenotype or transmission suggest a syndromic MID due to a mtDNA point mutation, DNA-microarrays using allele-specific oligonucleotide hybridisation, realtime-PCR or single-gene-sequencing are indicated. If the phenotype suggests a mLDNA deletion, RFLP or Southern-blot is indicated. For single or multiple mtDNA deletions with low heteroplasmy rate a long-range PCR may be helpful. If neither a single deletion nor multiple deletions are found, mtDNA sequencing is recommended. If multiple mtDNA deletions (breakage syndromes) are detected, sequencing of appropriate nDNA genes should be carried out. Sequencing should start with the POLG1 gene, since it is the one most frequently mutated. If the phenotype suggests a specific condition, such as GRACILE, Barth syndrome, Mohr-Tranebjaerg syndrome or MNGIE, appropriate nDNA genes need to be sequenced.

In case of a non-syndromic MID, biochemical investigations of the most affected tissue should clarify if a single or multiple RCCs are defective. In case of a single defect, sequencing of appropriate structural RCC-subunits or assembly-factors of the affected RCC is necessary. If biochemical investigations suggest CoQ-deficiency, sequencing of genes involved in the coenzyme-Q biosynthesis is indicated. In case of a single RCC-defect with maternal inheritance, one should proceed with mtDNA-sequencing. If multiple biochemical defects are found and Southern-blot or quantitative PCR detects mtDNA-depletion, sequencing of genes causing depletion-syndromes is recommended. If Southern-blot fails to detect mtDNA-depletion, sequencing of genes involved in the mitochondrial protein synthesis machinery (translation defects, elongation disorders) is indicated. since phenotypes associated with large-scale mtDNA rearrangements can be easily missed in leukocytes and the heteroplasmy of mtDNA mutations is often highest in skeletal muscle, investigations of muscle tissue is inevitable in these cases. Genetic testing is the gold standard for diagnosing MIDs and should be performed according to the phenotype, transmission or biochemical defect.

\section{Conclusions}

Since the field of clinical human neurogenetics is rapidly evolving, guidelines can only reflect the momentous state of knowledge and need to be revised regularly. Due to the expansion in the number of genetic tests actually or potentially available, it will be important to consider the impact of their costs in future analyses or to invent cheaper and more accurate tests. It is also important to note, on the other hand, that knowledge of the pathophysiology in rare neurogenetic disorders has already had a profound impact on more frequent sporadic diseases. Examining the molecular mechanisms can be more easily done in disorders, in which the precise cause is known at the genetic level. For this reason, the continuous effort in the search for the genetic background in families with neurogenetic disorders of yet unknown cause is inevitable. 
1. Gasser T, Dichgans M, Finsterer J, et al., EFNS Task Force on Molecular Diagnosis of Neurologic Disorders, Eur J Neurol, 2001;8:299-314.

2. Gasser T, Dichgans M, Finsterer J, et al., EFNS Task Force on Molecular Diagnosis of Neurologic Disorders: guidelines for the molecular diagnosis of inherited neurologic diseases. Second of two parts, Eur J Neurol, 2001;8:407-24.

3. Harbo HF, Finsterer J, Baets J, et al., EFNS guidelines on the molecular diagnosis of neurogenetic disorders: general issues, Huntington's disease, Parkinson's disease and dystonias, Eur J Neurol, 2009;16:777-85.

4. Gasser T, Finsterer J, Baets J, et al., EFNS guidelines on the molecular diagnosis of ataxias and spastic paraplegias, Eur J Neurol, 2010;17:179-88.

5. Finsterer J, Harbo HF, Baets J, et al., EFNS guidelines on the molecular diagnosis of mitochondrial disorders, Eur J Neurol, 2009;16:1255-64.

6. Burgunder JM, Schols L, Baets J, et al., EFNS guidelines for the molecular diagnosis of neurogenetic disorders: motoneuron, peripheral nerve and muscle disorders, Eur J Neurol, 2010 [Epub ahead of print].

7. Burgunder JM, Finsterer J, Szolnoki Z, et al., EFNS guidelines on the molecular diagnosis of channelopathies, epilepsies, migraine, stroke, and dementias, Eur J Neurol, 2010;17:641-8.

8. Brainin M, Barnes M, Baron JC, et al., Guidance for the preparation of neurological management guidelines by EFNS scientific task forces - revised recommendations 2004, Eur J Neurol, 2004;11:577-81.

9. Novak MJ, Tabrizi SJ, Huntington's disease, BMJ, 2010;340:C3109

10. The Huntington's Disease Collaborative Research Group A novel gene containing a trinucleotide repeat that is expanded and unstable on Huntington's disease chromosomes, Cell, 1993;26:971-83.

11. Guidelines for the molecular genetics predictive test in Huntington's disease. IHA and the WFN Research Group on Huntington's Chorea, Neurology, 1994;44:1533-6.

12. Wild EJ, Mudanohwo EE, Sweeney MG, et al., Huntington's disease phenocopies are clinically and genetically heterogeneous, Mov Disord, 2008;23:716-20.

13. Polymeropoulos MH, Lavedan C, Leroy E, et al., Mutation in the alpha-synuclein gene identified in famiies with Parkinson's disease, Science, 1997;276:2045-7.

14. Chartier-Harlin MC, Kachergus J, Roumier C, et al., Alpha-synuclein locus duplication as a cause of familial Parkinson's disease, Lancet, 2004;364:1167-9.

15. Singleton AB, Farrer $M$, Johnson J, et al., alpha-Synuclein locus triplication causes Parkinson's disease, Science, 2003;302:841.

16. Gasser T, Molecular pathogenesis of Parkinson disease: insights from genetic studies, Expert Rev Mol Med, 2009;11: e22.

17. Berg D, Niwar M, Maass S, et al., Alpha-synuclein and Parkinson's disease: implications from the screening of more than 1,900 patients, Mov Disord, 2005;20:1191-4.

18. Ibanez $P$, Lesage $S$, Janin $S$, et al., Alpha-synuclein gene rearrangements in dominantly inherited parkinsonism: frequency, phenotype, and mechanisms, Arch Neurol, 2009;66:102-8.

19. Berg D, Schweitzer K, Leitner P, et al., Type and frequency of mutations in the LRRK2 gene in familial and sporadic Parkinson's disease, Brain, 2005;128:3000-11.

20. Gilks WP, Abou-Sleiman PM, Gandhi S, et al., A common LRRK2 mutation in idiopathic Parkinson's disease, Lancet, 2005;365:415-16.

21. Ozelius $L$, Senthil G, Saunders-Pullman R, et al., LRRK2 G2019S as a cause of Parkinson's disease in Ashkenazi Jews, N Engl J Med, 2006;354:424-5.

22. Klein C, Schneider SA, Lang AE, Hereditary parkinsonism: Parkinson disease look-alikes - an algorithm for clinicians to 'PARK' genes and beyond, Mov Disord, 2009:24:2042-58.

23. Schmidt $\mathrm{A}$, Klein $\mathrm{C}$, The role of genes in causing dystonia, Eur J Neurol, 2010;17(Suppl. 1):65-70.

24. Kramer PL, Heiman GA, Gasser T, et al., The DYT1 gene on 9 q34 is responsible for most cases of early limb-onset idiopathic torsion dystonia in non-Jews, Am J Hum Genet, 1994;55:468-75.
25. Ozelius LJ, Hewett JW, Page CE, et al., The early-onset torsion dystonia gene (DYT1) encodes an ATP-binding protein, Nat Genet, 1997;17:40-8.

26. Ichinose $\mathrm{H}$, Ohye $\mathrm{T}$, Takahashi $\mathrm{E}$, et al., Hereditary progressive dystonia with marked diurnal fluctuation caused by mutations in the GTP cyclohydrolase I gene see comments, Nat Genet, 1994;8:236-42.

27. Zimprich A, Grabowski M, Asmus F, et al., Mutations in the gene encoding epsilon-sarcoglycan cause myoclonus-dystonia syndrome, Nat Genet, 2001;29:66-9.

28. Tanzi RE, Petrukhin K, Chernov I, et al., The Wilson disease gene is a copper transporting ATPase with homology to the Menkes disease gene, Nat Genet, 1993;5:344-50.

29. Soong BW, Paulson $\mathrm{HL}$, Spinocerebellar ataxias: an update, Curr Opin Neurol, 2007;20:438-46.

30. Moseley ML, Benzow KA, Schut $\sqcup$, et al., Incidence of dominant spinocerebellar and Friedreich triplet repeats among 361 ataxia families, Neurology, 1998;51:1666-71.

31. Silveira I, Miranda C, Guimaraes L, et al., Trinucleotide repeats in 202 families with ataxia, Arch Neurol, 2002;59: 623-9.

32. Brusco A, Gellera C, Cagnoli C, et al., Molecular genetics of hereditary spinocerebellar ataxia: mutation analysis of spinocerebellar ataxia genes and CAG/CTG repeat expansion detection in 225 Italian families, Arch Neurol, 2004;61:727-33.

33. Fogel BL, Perlman S, Clinical features and molecular genetics of autosomal recessive cerebellar ataxias, Lancet Neurol, 2007;6:245-57.

34. Pandolfo M, Friedreich ataxia, Semin Pediatr Neurol, 2003; 10:163-72

35. Taylor AM, Byrd PJ, Molecular pathology of ataxia telangiectasia, I Clin Pathol, 2005;58:1009-15.

36. Le Ber I, Brice A, Durr A, New autosomal recessive cerebellar ataxias with oculomotor apraxia, Curr Neurol Neurosci Rep, 2005;5:411-7.

37. Salinas S, Proukakis C, Crosby A, Warner $\Pi$, Hereditary spastic paraplegia: clinical features and pathogenetic mechanisms, Lancet Neurol, 2008;7:1127-38.

38. Patrono C, Scarano V, Cricchi F, et al., Autosomal dominant hereditary spastic paraplegia, Hum Mutat, 2005;25:506

39. Durr A, Camuzat A, Colin E, et al., Atlastin1 mutations are frequent in young-onset autosomal dominant spastic paraplegia, Arch Neurol, 2004;61:1867-72.

40. Woodward KJ, The molecular and cellular defects underlying Pelizaeus-Merzbacher disease, Expert Rev Mol Med, 2008;10:e14

41. AGS, Genetic testing for late-onset Alzheimer's disease AGS Ethics Committee, J Am Geriatr Soc, 2001;49:225-6.

42. Theuns J, Marjaux E, Vandenbulcke M, et al., Alzheimer dementia caused by a novel mutation located in the APP C-terminal intracytosolic fragment, Hum Mutat, 2006;27: $888-96$.

43. Brouwers N, Sleegers K, Van Broeckhoven C, Molecular genetics of Alzheimer's disease: an update, Ann Med, 2008;40:562-83.

44. Le Ber I, van der Zee J, Hannequin D, et al. Progranulin null mutations in both sporadic and familial frontotemporal dementia, Hum Mutat, 2007;28: 846-55.

45. Goldman JS, Farmer JM, Wood EM, et al., Comparison of family histories in FTLD subtypes and related tauopathies, Neurology, 2005;65:1817-9.

46. Markus HS, Unravelling the genetics of ischaemic stroke, PLOS Med, 2010;7:e1000225.

47. Matarin M, Singleton A, Hardy J, Meschia J, The genetics of ischaemic stroke, J Intern Med. 2010;267:139-55.

48. Chabriat $H$, Joutel A, Dichgans M, et al., Cadasil, Lancet Neurol, 2009;8:643-53.

49. Pantoni L, Cerebral small vessel disease: from pathogenesis and clinical characteristics to therapeutic challenges, Lancet Neurol, 2010;9:689-701.

50. Labauge $P$, Krivosic $V$, Denier $C$, et al., Frequency of retinal cavernomas in 60 patients with familial cerebral cavernomas, Arch Ophthalmol, 2006:124:885-6.

51. Platt $D$, Griggs $R$, Skeletal muscle channelopathies: new insights into the periodic paralyses and non-dystrophic myotonias, Curr Opin Neurol, 2009;22:524-31.
52. Baulac $S$, Baulac $M$, Advances on the genetics of mendelian idiopathic epilepsies, Clin Lab Med, 2010;30: 911-29.

53. Meisler MH, O'Brien JE, Sharkey LM, Sodium channel gene family: epilepsy mutations, gene interactions and modifier effects, J Physiol, 2010;588:1841-8.

54. de Vries B, Frants RR, Ferrari MD, van den Maagdenberg AM, Molecular genetics of migraine, Hum Genet, 2009;126:115-32.

55. Martyn $\mathrm{CN}$, Hughes RA, Epidemiology of periphera neuropathy, J Neurol Neurosurg Psychiatry, 1997;62: 310-18.

56. Dubourg O, Azzedine $\mathrm{H}$, Verny $\mathrm{C}$, et al., Autosomalrecessive forms of demyelinating Charcot-Marie-Tooth disease, Neuromolecular Med, 2006;8:75-86.

57. Szigeti K, Nelis E, Lupski JR, Molecular diagnostics of Charcot-Marie-Tooth disease and related peripheral neuropathies, Neuromolecular Med, 2006;8:243-54.

58. Schymick JC, Talbot K, Traynor BJ, Genetics of sporadic amyotrophic lateral sclerosis, Hum Mol Genet, 2007;16: R233-42.

59. Bushby K, Finkel R, Birnkrant DJ, et al., Diagnosis and management of Duchenne muscular dystrophy, part 1 Lancet Neurol, 2010;9:77-93.

60. Padberg GW, van Engelen BG, Facioscapulohumeral muscular dystrophy, Curr Opin Neurol, 2009;22:539-42.

61. Turner $\mathrm{C}$, Hilton-Jones $\mathrm{D}$, The myotonic dystrophies: diagnosis and management, I Neurol Neurosurg Psychiatry, 2010;81:358-67.

62. Bushby $K$, Diagnosis and management of the limb girdle muscular dystrophies, Pract Neurol, 2009;9:314-23.

63. McFarland R, Taylor RW, Turnbull DM, A neurological perspective on mitochondrial disease, Lancet Neurol, 2010;9:829-40.

64. Wu YT, Wu SB, Lee WY, Wei YH, Mitochondrial respiratory dysfunction-elicited oxidative stress and posttranslational protein modification in mitochondrial diseases, Ann N Y Acad Sci, 2010;1201:147-56.

65. Tang $\mathrm{S}$, Huang T, Characterization of mitochondrial DNA heteroplasmy using a parallel sequencing system, Biotechniques, 2010;48:287-96.

66. Zeviani M, Moraes $\mathrm{CT}$, DiMauro $\mathrm{S}$, et al., Deletions of mitochondrial DNA in Kearns-Sayre syndrome, Neurology, 1988;38:1339-46.

67. Holt IJ, Harding AE, Morgan-Hughes JA, Deletions of muscle mitochondrial DNA in patients with mitochondrial myopathies, Nature, 1988;331:717-9.

68. Rotig A, Colonna M, Bonnefont JP, et al., Mitochondrial DNA deletion in Pearson's marrow/pancreas syndrome, Lancet, 1989;1:902-3.

69. Wallace DC, singh G, Lott MT, et al., Mitochondrial DNA mutation associated with Leber's hereditary optic neuropathy, Science, 1988;242:1427-30.

70. Holt IJ, Harding AE, Petty RK, Morgan-Hughes JA, A new mitochondrial disease associated with mitochondrial DNA heteroplasmy, Am J Hum Genet, 1990;46:428-33.

71. Tatuch Y, Christodoulou J, Feigenbaum A, et al., Heteroplasmic mtDNA mutation ( $(-\mathrm{G}$ ) at 8993 can cause Leigh disease when the percentage of abnormal mtDNA is high, Am J Hum Genet, 1992;50:852-8.

72. Goto Y, Horai S, Matsuoka T, et al., Mitochondrial myopathy, encephalopathy, lactic acidosis, and stroke-like episodes (MELAS), Neurology, 1992;42: 545-50.

73. Shoffner JM, Lott MT, Lezza AM, et al., Myoclonic epilepsy and ragged-red fiber disease (MERRF) is associated with a mitochondrial DNA tRNA(Lys) mutation, Cell, 1990;61:931-7.

74. Nishino I, Spinazzola A, Hirano M, Thymidine phosphorylase gene mutations in MNGIE, a human mitochondrial disorder, Science, 1999;283:689-92.

75. Alexander $\mathrm{C}$, Votruba M, Pesch UE, et al., OPA1 encoding a dynamin-related GTPase, is mutated in autosomal dominant optic atrophy linked to chromosome 3q28, Nat Genet, 2000;26:211-5.

76. Hakonen AH, Goffart S, Marjavaara S, et al., Infantile-onset spinocerebellar ataxia and mitochondrial recessive ataxia syndrome are associated with neurona complex I defect and mtDNA depletion, Hum Mol Genet, 2008;17:3822-35 